\title{
A bibliometric analysis of acute respiratory distress syndrome (ARDS) research from 2010 to 2019
}

\author{
Xinyu Zhang ${ }^{1}$, Chengyuan Wang', Hongwen Zhao ${ }^{1}$ \\ ${ }^{1}$ Department of Pulmonary Critical Care Medicine, The First Hospital of China Medical University, Shenyang, China; ${ }^{2}$ Department of Urology, \\ The First Hospital of China Medical University, Shenyang, China \\ Contributions: (I) Conception and design: X Zhang, H Zhao; (II) Administrative support: X Zhang, C Wang; (III) Provision of study materials or \\ patients: C Wang; (IV) Collection and assembly of data: X Zhang, C Wang; (V) Data analysis and interpretation: X Zhang, C Wang; (VI) Manuscript \\ writing: All authors; (VII) Final approval of manuscript: All authors. \\ Correspondence to: Hongwen Zhao. Department of Pulmonary Critical Care Medicine, The First Hospital of China Medical University, 155 Nanjing \\ North Street, Heping District, Shenyang 110001, China. Email: hwzhao2007@163.com.
}

Background: Thousands of papers on acute respiratory distress syndrome (ARDS) have been published in the last decade. This study aimed to evaluate the research hotspots and future trends in ARDS research using bibliometric analysis.

Methods: All relevant literature on ARDS published between 2010 and 2019 was retrieved from the Web of Science Core Collection database, and the retrieval strategy was TS = (ARDS OR acute respiratory distress syndrome). Bibliometric analysis was conducted using VOSviewer and the online bibliometric analysis platform based on retrieved data. Bibliographic Item Co-occurrence Matrix Builder (BICOMB) and gCLUTO software were used to evaluate and visualize the results, and to explore the hotspots in the field of ARDS.

Results: A total of 9,858 ARDS research articles dated between 2010 and 2019 were included. The dominant position of the United States in global ARDS research throughout this 10 -year period was evident, and it was also the country most frequently involved in international cooperation. The University of Toronto was the most productive institution and a leader in research collaboration. Critical Care Medicine was the most productive journal in terms of the number of publications on ARDS. Further, Matthay MA, Pelosi P, Slutsky AS, and Thompson BT all made significant contributions to ARDS research. A total of 37 most frequent keywords were identified and belonged to 5 hotspots: (I) adult and pediatric ARDS; (II) life-support monitoring parameters and therapy in severe patients with ARDS; (III) molecular mechanisms of acute lung injury; (IV) influenza-related pneumonia; and (V) severe complications of ARDS. Also, in the last 5 years, the keywords "biomarkers", "pathway", "NF-кB", "epidemiology", "life-support", and "ECMO” began to appear in the ARDS research field.

Conclusions: In the decade from 2010 to 2019, the United States was a global leader in ARDS research, and hotspots included epidemiology, mechanisms, monitoring parameters, and therapy, especially mechanical ventilation. Our results suggest that the mechanisms of ARDS and novel life-support therapies will remain research hotspots in the future. International collaboration is also expected to widen and deepen in the field of ARDS research.

Keywords: Acute respiratory distress syndrome (ARDS); bibliometric analysis; VOSviewer; hotspots

Submitted Oct 16, 2020. Accepted for publication Jan 14, 2021.

doi: 10.21037/apm-20-2050

View this article at: http://dx.doi.org/10.21037/apm-20-2050 


\section{Introduction}

Acute respiratory distress syndrome (ARDS) is a lifethreatening clinical syndrome characterized by acute respiratory failure, refractory hypoxemia, and non-cardiogenic pulmonary edema $(1,2)$. ARDS affects $10.4 \%$ of all patients in intensive care units across the globe, and is associated with high risks of morbidity and mortality $(1,3,4)$. There are numerous risk factors associated with the development of ARDS, including pneumonia, sepsis, aspiration of gastric contents, major trauma, pulmonary contusion, inhalational injury, acute pancreatitis, severe burns, non-cardiogenic shock, multiple transfusions or transfusion-associated acute lung injury, drug overdose, pulmonary vasculitis, and drowning $(5,6)$. ARDS imposes an immense disease burden on patients and, being costly to treat, consumes a large proportion of health care resources $(7,8)$. ARDS was first described by Ashbaugh and colleagues in 1967 (9). During the past 50 years, especially in the last decade, great progress has been made in the field of ARDS research regarding epidemiology, diagnostics, pathology, pathophysiology, and therapeutics $(2,10-13)$. However, there is still a lack of comprehensive reports that can assist researchers in obtaining an intuitive overview and reveal research trends in the ARDS research field.

Bibliometric analysis is a novel scientific method used to evaluate contributions to a research field, including those by countries, institutions, authors, and journals. Further, bibliometric analysis can predict the hotspots and trends within a certain research area through information visualization (14-16). However, few bibliometric studies have been performed in the field of ARDS research.

In the present study, we performed a comprehensive bibliometric analysis of ARDS research literature from 2010 to 2019, taking into account the number of annual publications, countries, international cooperation, institutions, journals, authors, and keyword co-occurrence visualization analysis. Furthermore, perspectives on progress in the field of ARDS research over the past decade were considered. Overlay visualization maps of cooccurring keywords and double-clustering analysis were also performed in order to confirm the trends and hotspots in ARDS research. We hope that this study will provide new perspectives and a basis for future ARDS research.

\section{Methods}

\section{Data sources and search strategy}

The Web of Science is one of the most influential databases of scientific literature. In this study, all data were retrieved from the Web of Science Core Collection (WoSCC) via the China Medical University library website. The retrieval strategy was TS $=($ ARDS OR acute respiratory distress syndrome).

\section{Screening criteria and data downloads}

The publication period in the present study was limited to the period from 2010 to 2019. Non-English language, non-article, and non-review publications were excluded. WoSCC data including titles, author information, abstracts, keywords, journals, and references were downloaded in .txt format. To avoid the bias caused by frequent database updates, all literature retrieval and data downloads were completed on the same day (August 9, 2020). Two investigators (Xinyu Zhang and Chengyuan Wang) independently performed the search and had an agreement of $98 \%$ [kappa $=(\mathrm{P} 0-\mathrm{Pe}) /(\mathrm{n}-\mathrm{Pe})=0.98>0.75]$, showing significant consistency (17).

\section{Statistical analysis}

In the present study, a comprehensive description of various publishing characteristics is provided, including authors, institutions, countries, journals, keywords, impact factor (IF), and Hirsch index (h-index). IFs were obtained from the 2019 Journal Citation Reports (JCR) to assess the scientific value of research (18). The value of the h-index was defined as the number of papers with citation number $\geq \mathrm{h}$ and is considered to be an important indicator for assessing both the productivity and impact of the published work of scientists, journals, or countries (19). The filtered data from WoSCC was imported into the online analysis platform of literature metrology (http://bibliometric.com/) and VOSviewer 1.6.15 (Leiden University, Leiden, The Netherlands) for bibliometric analysis. Apache ECharts (https://echarts.apache.org/), a JavaScript-based data visualization tool, was used to visualize the annual number of publications and the number of cumulative publications in different countries/regions. The online bibliometric analysis platform was used to visualize international collaboration between countries. VOSviewer was used for analysis and visualization of bibliometric networks such as authors, institutions, journals, co-citations, and the keywords used in the articles (20). Network visualization maps and overlay visualization maps were generated using VOSviewer. The online bibliometric analysis platform 


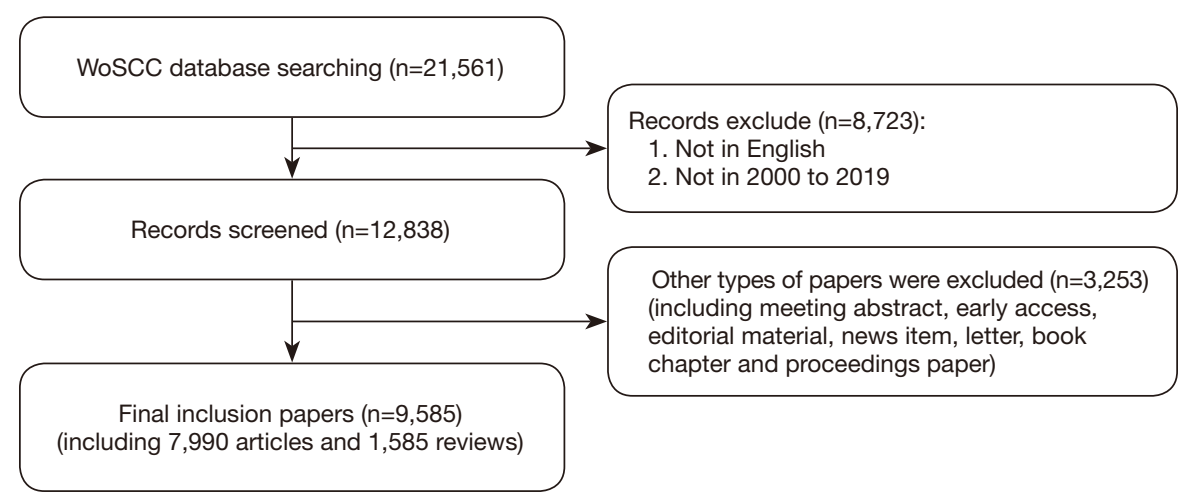

Figure 1 Flowchart of data filtration processing and excluding publications. WoSCC, Web of Science Core Collection.

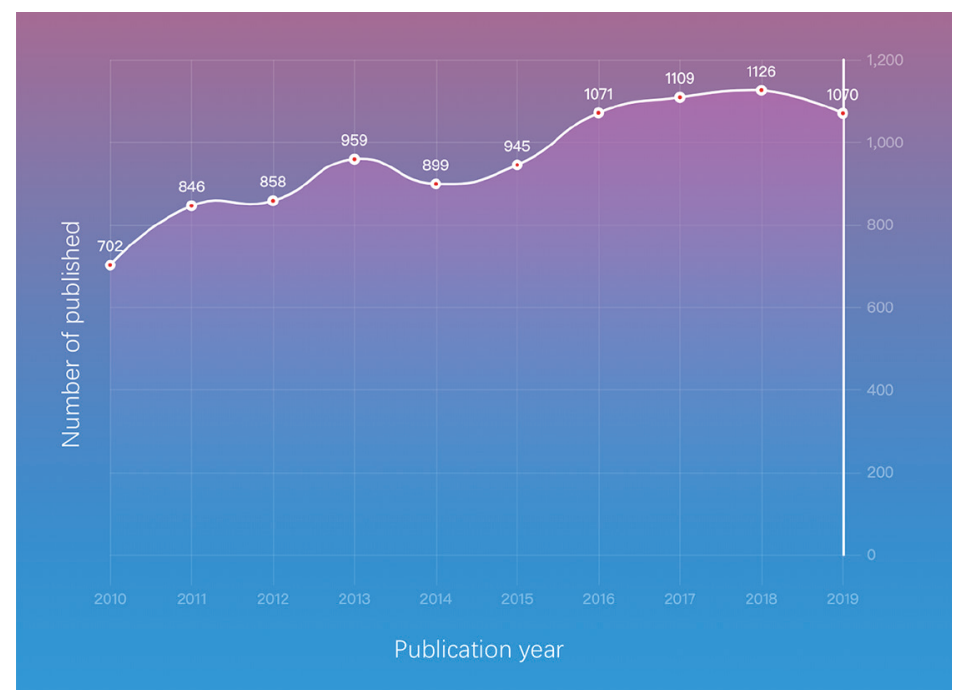

Figure 2 Annual number of the published publications in ARDS research from 2010 to 2019. ARDS, acute respiratory distress syndrome.

and Microsoft Excel 2016 were used to assess the impact of authors, institutions, and journals. The filtered data from WoSCC were imported into Bibliographic Item Co-occurrence Matrix Builder (BICOMB) to construct a keyword-article binary matrix (21). The rows of the matrix represented publications, while the columns represented highly frequent keywords. Additionally, gCLUTO software 1.0 was used to perform double-clustering analysis, and to build mountain maps and heat maps based on the results of the clustering analysis (21).

\section{Results}

\section{Trends and annual publications}

As shown in Figure 1, a total of 21,561 papers were identified, and 9,585 papers (7,990 articles and 1,595 reviews) from 2010 to 2019 were ultimately included according to the screening criteria. Figure 2 shows the growth trend of the annual publications related to ARDS, from 702 in 2010 to 1,070 in 2019. Based on the WoSCC database, the 9,585 papers were cited 211,730 times, and each paper was cited an average of 22.09 times.

\section{Contribution of countries and institutions}

According to the WoSCC database, 117 countries or regions contributed to publications on ARDS between 2010 and 2019. The top 22 countries or regions in terms of the number of publications $(\mathrm{n} \geq 60)$ on ARDS are presented on a world map in Figure $3 A$, and the top 10 are presented 
A

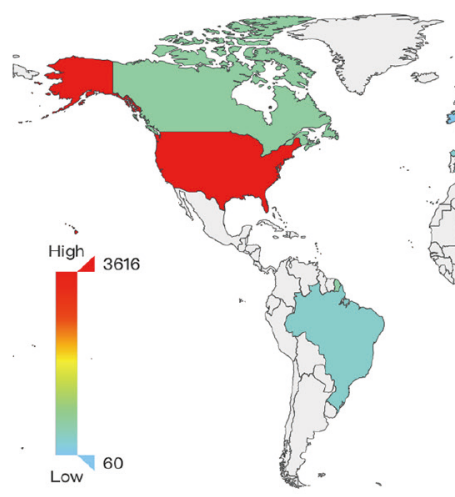

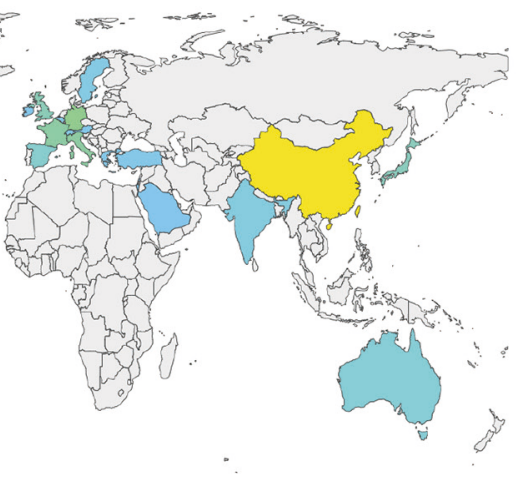

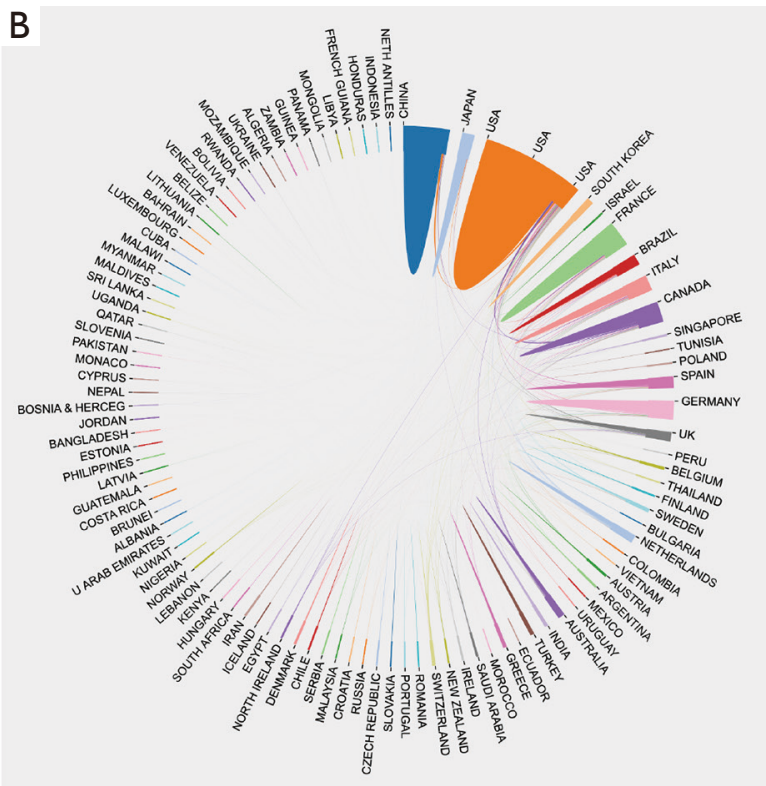

Figure 3 The distribution of countries or regions in ARDS research. (A) Distribution of ARDS literatures in the world map. The color of each country or region on the world map represents the amount of literature published, according to the color gradient in the lower left corner. (B) The network map of cooperation between countries or regions. Different colors represent different countries or regions, the area of each color represents the amount of literature published in each country or regions, and the thickness of the connecting line indicates the cooperation frequency. ARDS, acute respiratory distress syndrome.

Table 1 The top 10 countries or regions contributing to publications in ARDS research

\begin{tabular}{lccc}
\hline Rank & Country/region & Records & Percentage (N/9,585), \% \\
\hline 1 & USA & 3,616 & 37.726 \\
2 & China & 1,936 & 20.198 \\
3 & Germany & 868 & 9.056 \\
4 & Canada & 715 & 7.46 \\
5 & Italy & 697 & 7.272 \\
6 & France & 671 & 7.001 \\
7 & UK & 517 & 5.394 \\
8 & Japan & 465 & 4.851 \\
9 & Brazil & 372 & 3.881 \\
10 & Spain & 345 & 3.599 \\
\hline
\end{tabular}

ARDS, acute respiratory distress syndrome.

as numbers in Table 1. The United States was the largest contributor, with 3,616 papers published, followed by China ( $\mathrm{n}=1,936)$, Germany $(\mathrm{n}=868)$, Canada $(\mathrm{n}=715)$, Italy $(n=697)$, France $(n=671)$, the United Kingdom $(n=517)$,
Japan ( $n=465)$, Brazil $(n=372)$, and Spain $(n=345)$. The United States and China contributed many more papers to the number of publications on ARDS than other countries or regions (Figure $3 A$, Table 1). Within the survey period, close cooperation between countries or regions around the world was extremely common. International cooperation analysis indicated that the United States was the country most frequently involved in international cooperation (Figure 3B).

The most productive institutions were also evaluated in our study. As shown in Table 2, with 788 papers published, the University of Toronto was the most productive institution, and was followed by the University of California, San Francisco $(\mathrm{n}=656)$, Johns Hopkins University $(\mathrm{n}=422)$, University of Pittsburgh ( $\mathrm{n}=383)$, Mayo Clinic $(\mathrm{n}=372)$, University of Pennsylvania ( $\mathrm{n}=355)$, Vanderbilt University $(\mathrm{n}=306)$, University of Washington $(\mathrm{n}=299)$, University of Michigan ( $\mathrm{n}=284)$, and Harvard University $(\mathrm{n}=282)$. Among the top 10 most productive institutions, the University of Toronto was in Canada and the rest were from the United States. The collaboration network was generated using VOSviewer software, and the threshold was set to 66 as the minimum number of documents of an institution, while 
Table 2 The top 10 most productive institutions in ARDS research

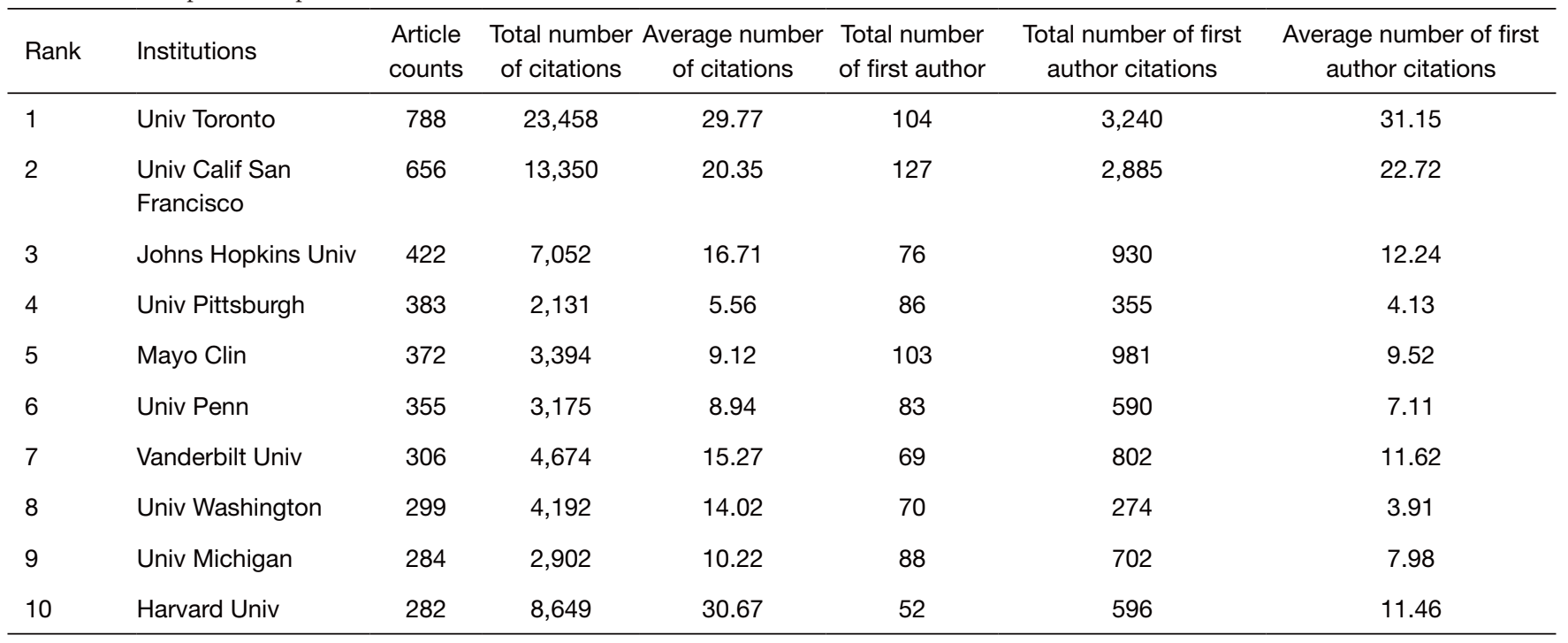

ARDS, acute respiratory distress syndrome.

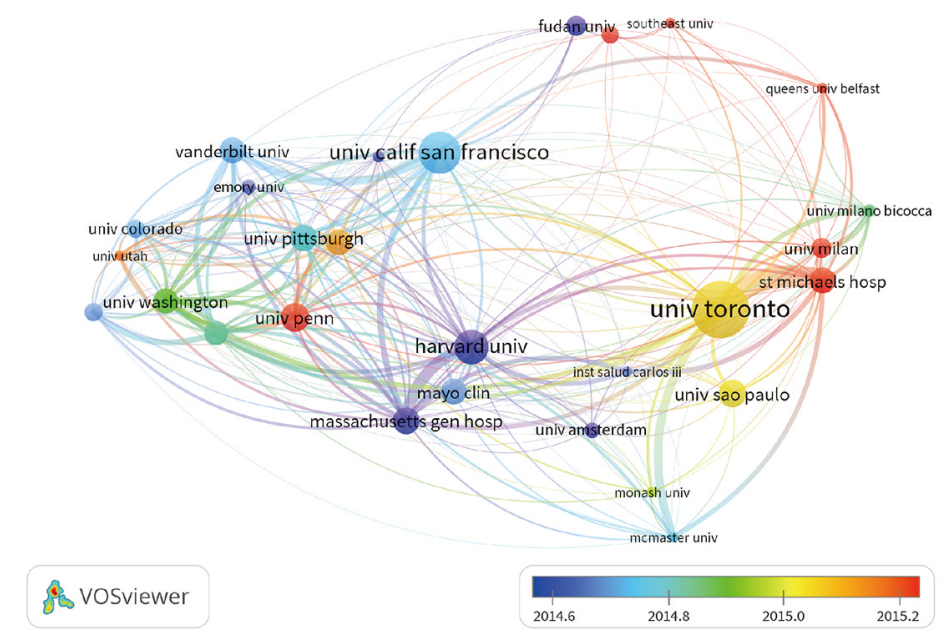

Figure 4 Co-authorship overlay visualization map of institutions. The color of each circle corresponds to the average publication year, the size of a circle is proportional to the number of literatures, and the thickness of the connecting line indicates the cooperation frequency.

2,000 was set as the minimum number of citations of an institution. Finally, 28 out of the 7,443 institutions were identified (Figure 4). During this decade, the University of Toronto published 788 papers, which were cited 23,458 times (Table 2), and cooperated with almost all influential scientific institutions in studies on ARDS (Figure 4).

\section{Contribution of journals}

In the present study, a comprehensive analysis of the contribution of journals with journal characteristics was provided, including journal titles, article counts, total citations, citations per article, CiteScore [2019], IF [2019], quartile in category [2019], and h-index. The top 10 most productive journals in the field of ARDS research are listed in Table 3; in total, these journals published 2,155 papers, accounting for $22.48 \%$ of the total publications. Critical Care Medicine ( $\mathrm{n}=384)$, Critical Care $(\mathrm{n}=321)$, and PloS One $(n=249)$ were the top 3 journals in terms of the number of publications on ARDS (Table 3). The American fournal of 
Table 3 The top 10 most active journals that published articles in ARDS research

\begin{tabular}{|c|c|c|c|c|c|c|c|c|}
\hline Rank & Journal title & $\begin{array}{l}\text { Article } \\
\text { counts }\end{array}$ & $\begin{array}{l}\text { Total number } \\
\text { of citations }\end{array}$ & $\begin{array}{l}\text { Average number } \\
\text { of citations }\end{array}$ & CiteScore & IF [2019] & $\begin{array}{c}\text { Quartile in } \\
\text { category [2019] }\end{array}$ & $\mathrm{H}$-index \\
\hline 1 & Critical Care Medicine & 384 & 4,199 & 10.93 & 10.6 & 7.414 & Q1 & 33 \\
\hline 3 & PLoS One & 249 & 848 & 3.41 & 5.2 & 2.74 & Q2 & NA \\
\hline 6 & $\begin{array}{l}\text { American Journal of Respiratory } \\
\text { and Critical Care Medicine }\end{array}$ & 188 & 4,494 & 23.9 & 21.6 & 17.452 & Q1 & 48 \\
\hline 7 & $\begin{array}{l}\text { American Journal of Physiology- } \\
\text { Lung Cellular and Molecular } \\
\text { Physiology }\end{array}$ & 177 & 954 & 5.39 & 7.2 & 4.406 & Q1 & 22 \\
\hline 10 & Shock & 118 & 456 & 3.86 & 6.2 & 2.96 & Q2 & 19 \\
\hline
\end{tabular}

ARDS, acute respiratory distress syndrome; IF, impact factor; H-index, Hirsch index; JCR, Journal Citation Reports.

Respiratory and Critical Care Medicine, Intensive Care Medicine, and Critical Care Medicine were the top 3 journals in terms of the highest total number of citations $(4,494 v s .4,203 v s$. 4,199 citations, respectively), and they were also the top 3 journals with the highest average number of citations per paper (23.9 vs. 19.92 vs. 10.93 times, respectively). Intensive Care Medicine, the American Fournal of Respiratory and Critical Care Medicine, and Critical Care Medicine also had the highest IFs of any journals in 2019 (17.679 vs. $17.452 v s$. 7.414, respectively). The highest CiteScore and the highest h-index belonged to the American Fournal of Respiratory and Critical Care Medicine (21.67 and 48, respectively). Among the top 10 most productive journals, Critical Care Medicine, Critical Care, Intensive Care Medicine, the American fournal of Respiratory and Critical Care Medicine, and the American Fournal of Physiology-Lung Cellular and Molecular Physiology were classified as Q1 according to the JCR 2019 standards (Table 3). The top 10 most highly cited publications are listed in Table 4.

\section{Contributions of authors}

The top 10 most productive authors in the field of ARDS research are presented in Table 5. Among them, Matthay MA from the Department of Medicine and Anesthesia, Cardiovascular Research Institute, University of California, San Francisco in the United States ranked first $(n=149)$.
Pelosi P from the Department of Surgical Sciences and Integrated Diagnostics, University of Genoa in Italy was the second most productive author $(\mathrm{n}=104)$ (Table 5). Furthermore, Slutsky AS, Thompson BT, and Matthay MA were the top 3 authors with the highest total number of citations (5,055 vs. 5,038 vs. 3,736 times, respectively, Table 5). A co-authorship overlay visualization map was generated using VOSviewer software, and the threshold for the minimum number of documents by an author was set to 35 . Finally, 31 authors who met the threshold were identified, and Matthay MA, Thompson BT, Calfee CS, and Ware LB were shown to have cooperated closely (Figure 5A). A citation overlay visualization map was also generated using VOSviewer software, and the threshold for the minimum number of citations of an author was set to 2,000. Finally, 37 authors who met the threshold were identified, and it could be seen that Matthay MA, Pelosi P, Slutsky AS, and Thompson BT had made significant contributions to the field of ARDS research (Figure 5B and Table 5).

\section{Analysis of research hotspots}

With an appearance of more than 47 times, 37 of the most frequent keywords were extracted from the included publications and are displayed in Table 6. Five clusters were sorted through double-clustering using gCLUTO. The relationship between publications and high-frequency 
Table 4 The top 10 high-cited papers in ARDS research during 2010 to 2019

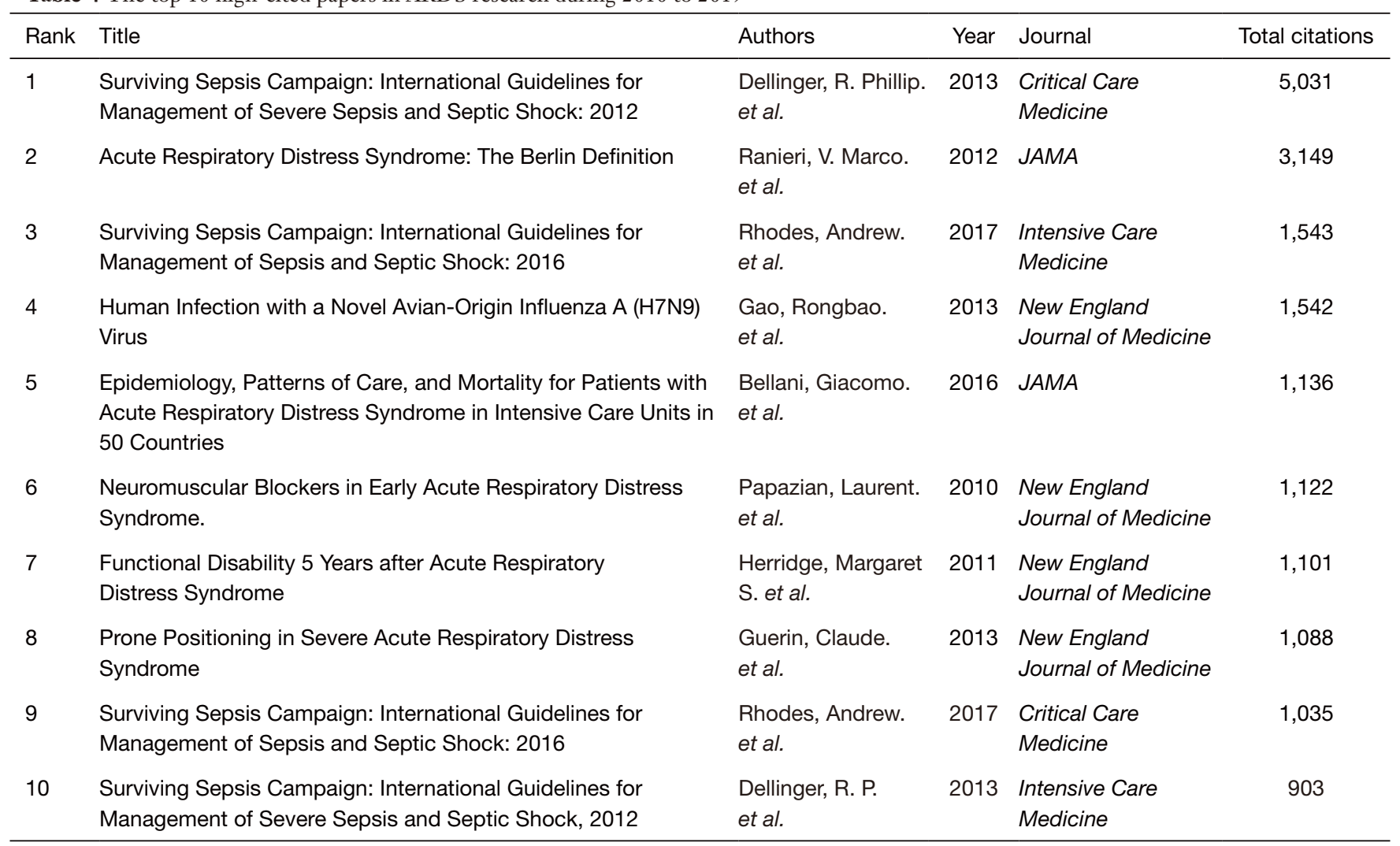

ARDS, acute respiratory distress syndrome.

Table 5 The top 10 most productive authors in ARDS research

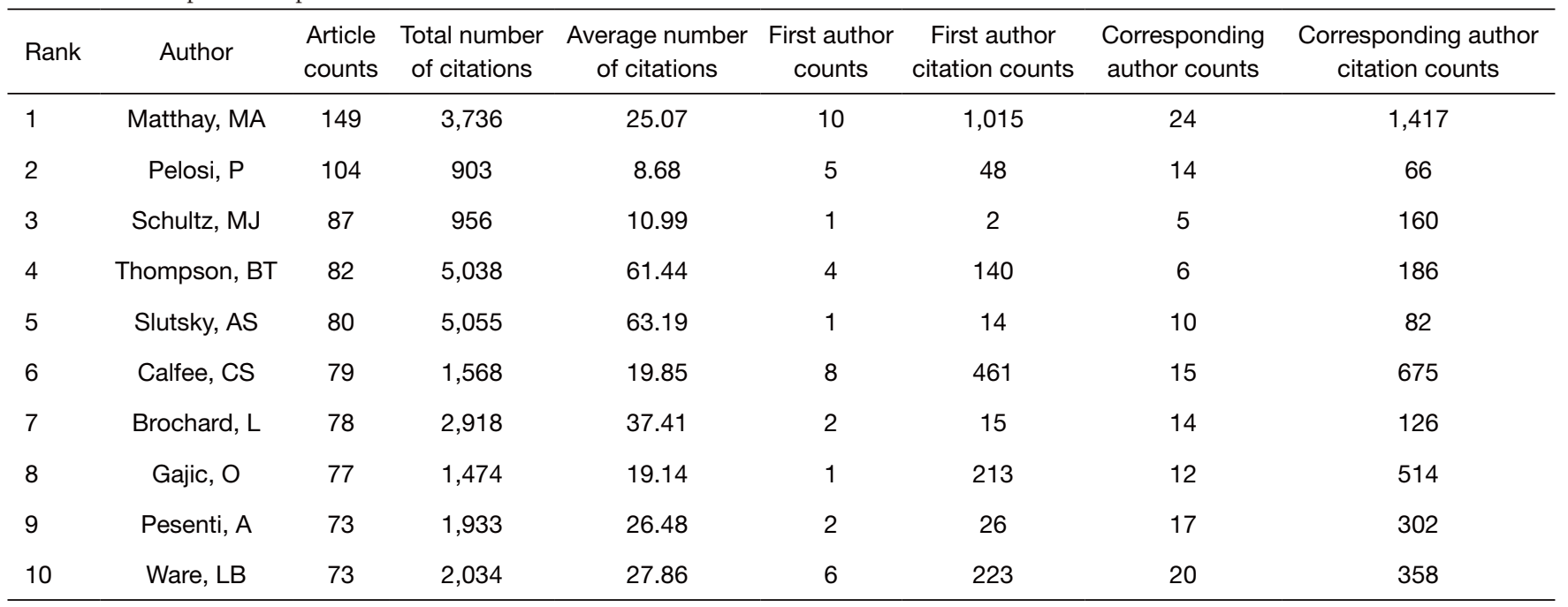

ARDS, acute respiratory distress syndrome. 
A

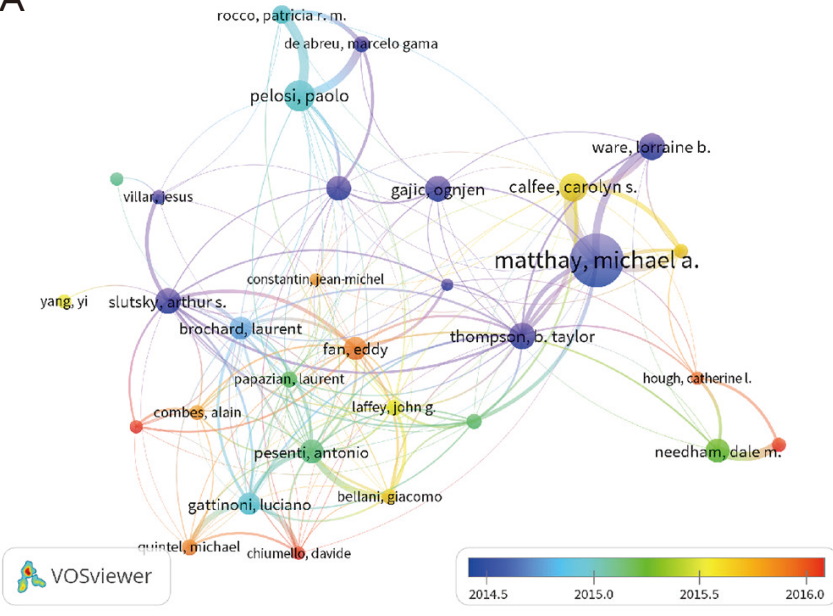

B

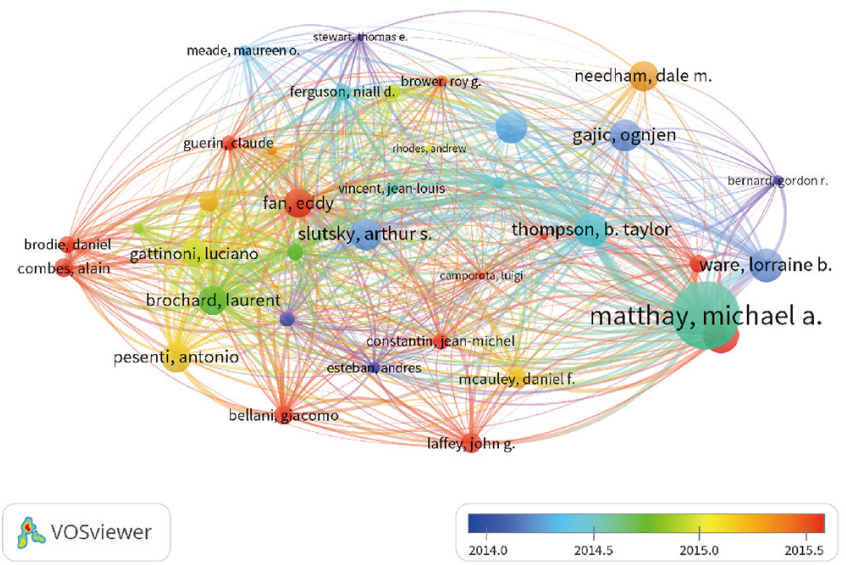

Figure 5 The distribution of authors in ARDS research. (A) Author co-authorship overlay visualization map. Notes: the color of each circle corresponds to the average publication year of the author, the size of a circle is proportional to the number of literatures published by the author, and the thickness of the connecting line indicates the cooperation frequency. (B) Author co-citation overlay visualization map. Notes: the color of each circle corresponds to the average publication year of the author, the size of a circle is proportional to the total number of citations of the author, and the thickness of the connecting line indicates the strength of the co-citation link. ARDS, acute respiratory distress syndrome.

keywords was visualized using a volcano map and matrix map (Figure 6). The matrix map is shown in Figure 6A, in which column labels represent articles, while row labels represent keywords. To combine similar rows in a single cluster, the rows of the initial matrix were reset and each cluster was partitioned by black horizontal lines. In the matrix map, the upper dendrogram represents article associations, while the left represents high-frequency keyword associations. The results of the volcano map in Figure $6 B$ directly show the high-dimensional character of the data. In this 3-dimensional image, 5 different mountains represent 5 different clusters, numbered from 0 to 4 .

The above 37 high-frequency keywords were divided into 5 clusters. All representative articles involved in each cluster were mined to further summarize hotspots in the field of ARDS. Finally, 5 hotspots were identified using BICOMB and gCLUTO software packages:

* Cluster 0: adult and pediatric ARDS.

* Cluster 1: life-support monitoring parameters and therapy in severe patients with ARDS.

* Cluster 2: molecular mechanisms of acute lung injury.

* Cluster 3: influenza-related pneumonia.

* Cluster 4: severe complications of ARDS.

To explore the changes of hotspots over a period of time, a network visualization map of keyword co-occurrence was generated using VOSviewer software, and the results showed that the keywords "biomarkers", "pathway", "NFкB", "epidemiology", "life-support", and "ECMO" began to appear in the last 5 years (Figure 7).

\section{Discussion}

In the era of the information explosion, bibliometric analysis can help scientific researchers to manage their knowledge and visualize knowledge structures more intuitively. By presenting visual results, bibliometric analysis can help new researchers in a specific field to grasp the overall trends in the field being investigated. It can also reveal milestone manuscripts, the most productive authors and institutions, and current research hotspots, as well as future trends (22-24). In our study, a comprehensive bibliometric analysis of global scientific publications in the field of ARDS research from 2010 to 2019 was performed.

The number of publications in a particular research field can reflect the productivity and developments in the field over time (25). In the present study, a total of 9,585 publications, including 1,070 articles in 2019 , were included (Figures 1,2). The results showed that the number of publications in the field of ARDS was maintained at a 
Table 6 Keywords of ARDS research hotspots

\begin{tabular}{|c|c|c|c|c|}
\hline Number & Keywords & Frequency & Percent (\%) & Accumulate percent (\%) \\
\hline 2 & Acute lung injury & 1,334 & 4.2770 & 12.1513 \\
\hline 3 & Mechanical ventilation & 550 & 1.7634 & 13.9147 \\
\hline 4 & Extracorporeal membrane oxygenation & 415 & 1.3306 & 15.2453 \\
\hline 7 & Sepsis & 302 & 0.9683 & 18.4546 \\
\hline 8 & Lung injury & 222 & 0.7118 & 19.1664 \\
\hline 9 & Mortality & 221 & 0.7086 & 19.8750 \\
\hline 13 & Respiratory distress syndrome & 146 & 0.4681 & 21.8403 \\
\hline 14 & Acute respiratory failure & 142 & 0.4553 & 22.2956 \\
\hline 15 & Ventilator-induced lung injury & 131 & 0.4200 & 22.7156 \\
\hline 16 & Intensive care & 117 & 0.3751 & 23.0907 \\
\hline 17 & Apoptosis & 116 & 0.3719 & 23.4626 \\
\hline 18 & Cytokines & 111 & 0.3559 & 23.8185 \\
\hline 19 & Intensive care unit & 109 & 0.3495 & 24.1680 \\
\hline 20 & Lung & 102 & 0.3270 & 24.4950 \\
\hline 26 & Acute kidney injury & 76 & 0.2437 & 26.2264 \\
\hline 27 & Oxidative stress & 76 & 0.2437 & 26.4700 \\
\hline 28 & Prognosis & 72 & 0.2308 & 26.7009 \\
\hline 29 & Epidemiology & 69 & 0.2212 & 26.9221 \\
\hline 30 & Pediatrics & 66 & 0.2116 & 27.1337 \\
\hline 31 & Adult & 65 & 0.2084 & 27.3421 \\
\hline 32 & Pulmonary edema & 60 & 0.1924 & 27.5345 \\
\hline 33 & Septic shock & 57 & 0.1828 & 27.7172 \\
\hline 34 & Peep & 49 & 0.1571 & 27.8743 \\
\hline 35 & $\mathrm{H} 1 \mathrm{~N} 1$ & 48 & 0.1539 & 28.0282 \\
\hline 36 & Prone position & 48 & 0.1539 & 28.1821 \\
\hline 37 & Influenza & 47 & 0.1507 & 28.3328 \\
\hline
\end{tabular}

ARDS, acute respiratory distress syndrome. 

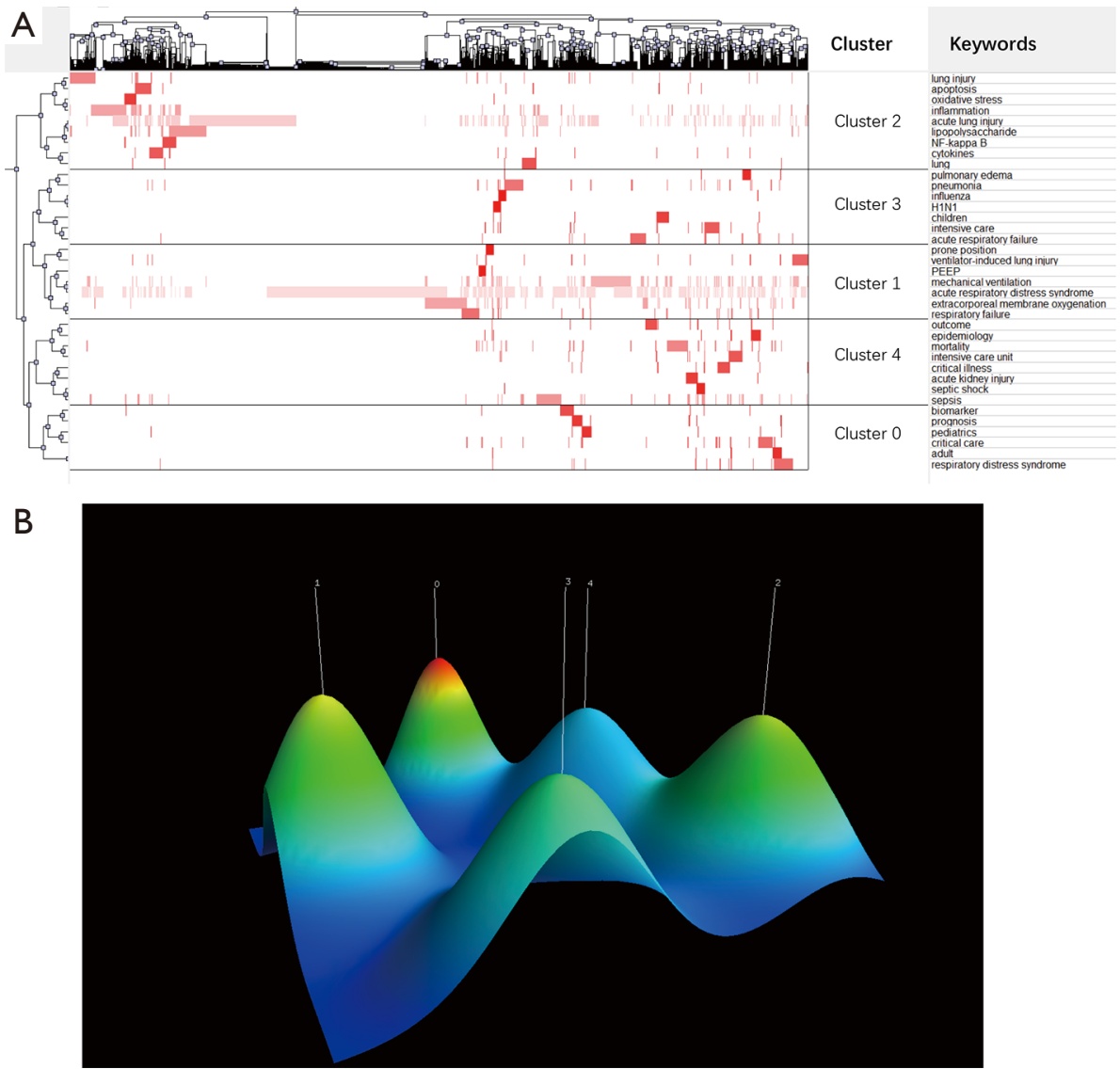

Figure 6 Research hotspots in the field of ARDS. (A) Visualized matrix of biclustering of highly frequent keywords in the research field of ARDS. Color of each blot represented the frequency of occurrence of keywords in all literatures. (B) Mountain visualization of biclustering of highly frequent keywords in the research field of ARDS. The height and color of the mountain is proportional to internal similarity and standard deviation of cluster (Blue: high deviation; Red: low deviation). ARDS, acute respiratory distress syndrome.

substantial level in the decade from 2010 to 2019, which suggests that ARDS remains a hot research field, and more and more scholars may participate in ARDS research.

The number of publications in a research field is an important indicator for evaluating the scientific research level of a country or institution $(22,25,26)$. Our study showed that the United States and China were the 2 largest contributors to the number of publications on ARDS (Figure $3 \mathrm{~A}$, Table 1), highlighting their impact in the ARDS research field. The value of international collaboration in supporting innovation and addressing unmet challenges is well recognized worldwide (27). From 2010 to 2019, many countries or regions around the world collaborated on studies in the research field of ARDS. Furthermore, our results demonstrated that the United States had the highest collaboration performance, especially with Canada and China (Figure 3B). Meanwhile, the University of Toronto was identified as the most productive institution, with the highest total citation number during the 10-year period (Table 2), and cooperated with almost all influential scientific institutions in the ARDS research field, including Harvard University and St. Michael's Hospital (Figure 4). These results showed that highly collaborative countries or institutions generally had a high academic level, suggesting that international cooperation will remain a future trend in the field of ARDS research.

Journal indicators obtained from bibliometric analysis can provide a reliable reference for researchers to search documents or submit manuscripts $(28,29)$. Our results showed that 8 of the top 10 journals publishing literature on ARDS were included in the category of "critical care medicine", while PLoS One was listed in the category of 


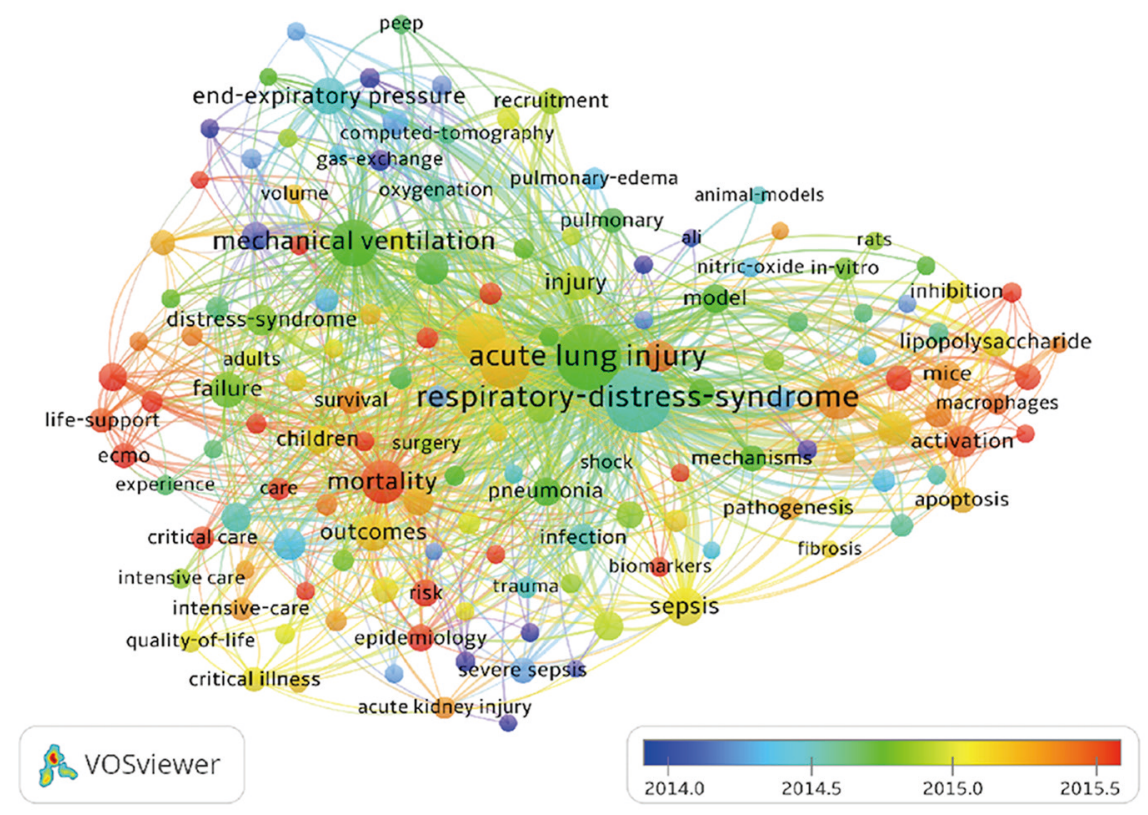

Figure 7 Keywords co-occurrence overlay visualization map. Notes: the color of each circle corresponds to the average publication year. The size of a circle is proportional to the frequency of occurrence of the keyword, and the thickness of the connecting line indicates the strength of the keywords co-occurrence link.

"multidisciplinary sciences" and the American fournal of Physiology-Lung Cellular and Molecular Physiology was listed in the category of "physiology". Critical Care Medicine published the highest number of ARDS-related papers, while the American Fournal of Respiratory and Critical Care Medicine had the highest number of total citations (Table 3), which suggests that ARDS plays an important role in the fields of critical care and intensive care medicine. Our results also showed that the most frequently cited publication was a practice guideline written by the Surviving Sepsis Campaign Guidelines Committee, and that "The Acute Respiratory Distress Syndrome: the Berlin Definition”, written by the ARDS Definition Task Force, was a milestone in the ARDS research field in the decade from 2010 to 2019 (1) (Table 4). These results suggest that these active journals and highly cited papers can provide a reliable reference for scholars concerned with the progress of ARDS research.

Based on the WoSCC database, Matthay MA published the highest number of ARDS-related papers, while Thompson BT had the highest number of total citations (Table 5). Furthermore, Matthay MA, Thompson BT, Slutsky AS, Brochard L, Ware LB, and Gajic O cooperated closely and published a considerable number of highly cited publications, as evidenced in the co-authorship overlay network visualization map and citation overlay visualization map (Figure $5 A, B$ ). Therefore, they can be regarded as the leaders in the ARDS research field.

Because of the heterogeneity of the ARDS research field, we divided the keywords in our study into 5 clusters via double-clustering analysis (Figure 6). Keyword cooccurrence network visualization analysis is a widely accepted method for determining research hotspots and predicting research trends (30). Our results indicated that the early stage of ARDS research was focused on severe sepsis and monitoring parameters. Subsequently, keywords such as "epidemiology", "life-support", "ECMO", "biomarker", "activation", and "mice" appeared frequently in the last 5 years, suggesting that the study of the mechanisms of ARDS and therapeutic strategies will remain research hotspots over the next few years. Our analysis also suggests that scientists are still trying to gain a comprehensive understanding of ARDS, and we expect scientists to make breakthroughs in the pathogenesis and treatment of this condition in the near future.

However, there were some limitations in our study. Firstly, the WoSCC database is updated continuously and dynamically. Therefore, our results are temporary in nature. 
Secondly, non-English publications were excluded. Hence, a discrepancy may exist between our results and the real publication characteristics.

In conclusion, the annual number of publications on ARDS grew in the decade between 2010 and 2019. The United States was the leading country in this research field, while the University of Toronto also achieved important research results and played a certain role in promoting the development of ARDS research. Furthermore, Matthay MA, Pelosi P, Slutsky AS, and Thompson BT made significant contributions to this research field. The hotspots over the decade were epidemiology, mechanisms, monitoring parameters, and therapy, especially mechanical ventilation. Our results suggest that the mechanisms of ARDS and novel life-support therapies will remain research hotspots in the future. International collaboration was also prevalent, and it is expected to widen and deepen in the future. These results provide new perspectives for the study of ARDS and may have a beneficial effect on further study regarding the etiology, diagnosis, and treatment of this condition.

\section{Acknowledgments}

Funding: Our study was funded by the Project of Liaoning Distinguished Professor [grant No. (2013) 204] to Hongwen Zhao.

\section{Footnote}

Conflicts of Interest: All authors have completed the ICMJE uniform disclosure form (available at http://dx.doi. org/10.21037/apm-20-2050). The authors have no conflicts of interest to declare.

Ethical Statement: The authors are accountable for all aspects of the work in ensuring that questions related to the accuracy or integrity of any part of the work are appropriately investigated and resolved.

Open Access Statement: This is an Open Access article distributed in accordance with the Creative Commons Attribution-NonCommercial-NoDerivs 4.0 International License (CC BY-NC-ND 4.0), which permits the noncommercial replication and distribution of the article with the strict proviso that no changes or edits are made and the original work is properly cited (including links to both the formal publication through the relevant DOI and the license). See: https://creativecommons.org/licenses/by-nc-nd/4.0/.

\section{References}

1. Force ADT, Ranieri VM, Rubenfeld GD, et al. Acute respiratory distress syndrome: the Berlin Definition. JAMA 2012;307:2526-33.

2. Matthay MA, Zemans RL, Zimmerman GA, et al. Acute respiratory distress syndrome. Nat Rev Dis Primers 2019;5:18.

3. Meyer NJ, Calfee CS. Novel translational approaches to the search for precision therapies for acute respiratory distress syndrome. Lancet Respir Med 2017;5:512-23.

4. Bellani G, Laffey JG, Pham T, et al. Epidemiology, Patterns of Care, and Mortality for Patients With Acute Respiratory Distress Syndrome in Intensive Care Units in 50 Countries. JAMA 2016;315:788-800.

5. Ware LB, Matthay MA. The acute respiratory distress syndrome. N Engl J Med 2000;342:1334-49.

6. Ferguson ND, Fan E, Camporota L, et al. The Berlin definition of ARDS: an expanded rationale, justification, and supplementary material. Intensive Care Med 2012;38:1573-82.

7. Herridge MS, Tansey CM, Matte A, et al. Functional disability 5 years after acute respiratory distress syndrome. N Engl J Med 2011;364:1293-304.

8. Bice T, Carson SS. Acute Respiratory Distress Syndrome: Cost (Early and Long-Term). Semin Respir Crit Care Med 2019;40:137-44.

9. Ashbaugh DG, Bigelow DB, Petty TL, et al. Acute respiratory distress in adults. Lancet 1967;2:319-23.

10. Papazian L, Calfee CS, Chiumello D, et al. Diagnostic workup for ARDS patients. Intensive Care Med 2016;42:674-85.

11. Matthay MA, Zemans RL. The acute respiratory distress syndrome: pathogenesis and treatment. Annu Rev Pathol 2011;6:147-63.

12. Howell MD, Davis AM. Management of ARDS in Adults. JAMA 2018;319:711-2.

13. Matthay MA. ECMO in severe acute respiratory distress syndrome. Lancet Respir Med 2019;7:106-8.

14. Cooper ID. Bibliometrics basics. J Med Libr Assoc 2015;103:217-8.

15. Devos P, Menard J. Trends in Worldwide Research in Hypertension Over the Period 1999-2018: A Bibliometric Study. Hypertension 2020;76:1649-55.

16. Wang $Y$, Zhao N, Zhang $X$, et al. Bibliometrics Analysis of Butyrophilins as Immune Regulators [1992-2019] and Implications for Cancer Prognosis. Front Immunol 2020;11:1187. 
17. Landis JR, Koch GG. The measurement of observer agreement for categorical data. Biometrics 1977;33:159-74.

18. Eyre-Walker A, Stoletzki N. The assessment of science: the relative merits of post-publication review, the impact factor, and the number of citations. PLoS Biol 2013;11:e1001675.

19. Hirsch JE. An index to quantify an individual's scientific research output. Proc Natl Acad Sci U S A 2005;102:16569-72.

20. van Eck NJ, Waltman L. Citation-based clustering of publications using CitNetExplorer and VOSviewer. Scientometrics 2017;111:1053-70.

21. Li F, Li M, Guan P, et al. Mapping publication trends and identifying hot spots of research on Internet health information seeking behavior: a quantitative and co-word biclustering analysis. J Med Internet Res 2015;17:e81.

22. Glanville J, Kendrick T, McNally R, et al. Research output on primary care in Australia, Canada, Germany, the Netherlands, the United Kingdom, and the United States: bibliometric analysis. BMJ 2011;342:d1028.

23. Boudry C, Baudouin C, Mouriaux F. International publication trends in dry eye disease research: A bibliometric analysis. Ocul Surf 2018;16:173-9.

Cite this article as: Zhang $\mathrm{X}$, Wang $\mathrm{C}$, Zhao $\mathrm{H}$. A bibliometric analysis of acute respiratory distress syndrome (ARDS) research from 2010 to 2019. Ann Palliat Med 2021;10(4):3750-3762. doi: 10.21037/apm-20-2050
24. Stout NL, Alfano CM, Belter CW, et al. A Bibliometric Analysis of the Landscape of Cancer Rehabilitation Research (1992-2016). J Natl Cancer Inst 2018;110:815-24.

25. Durieux V, Gevenois PA. Bibliometric indicators: quality measurements of scientific publication. Radiology 2010;255:342-51.

26. Soteriades ES, Falagas ME. Comparison of amount of biomedical research originating from the European Union and the United States. BMJ 2005;331:192-4.

27. Gal D, Glanzel W, Sipido KR. Mapping cross-border collaboration and communication in cardiovascular research from 1992 to 2012. Eur Heart J 2017;38:1249-58.

28. Oelrich B, Peters R, Jung K. A Bibliometric evaluation of publications in Urological journals among European Union countries between 2000-2005. Eur Urol 2007;52:1238-48.

29. Garfield E. Journal impact factor: a brief review. CMAJ 1999;161:979-80.

30. Chen C. Searching for intellectual turning points: progressive knowledge domain visualization. Proc Natl Acad Sci U S A 2004;101 Suppl 1:5303-10. 